\title{
Towards minimal models for realistic granular materials: Tomographic analysis of bidispersed assemblies of ellipsoids
}

\author{
Fabian M. Schaller ${ }^{1, \star}$, Horst Punzmann², Gerd E. Schröder-Turk ${ }^{3,1, \star \star}$, and Mohammad Saadatfar ${ }^{4, \star \star \star ~}$ \\ ${ }^{1}$ Institut für theoretische Physik, Friedrich-Alexander-Universität Erlangen-Nürnberg, Erlangen, Germany \\ ${ }^{2}$ Centre for Plasmas and Fluids, Research School of Physics and Engineering, Australian National University, Canberra ACT \\ 2601, Australia \\ ${ }^{3}$ School of Engineering and Information Technology, Murdoch University, Murdoch WA 6150, Australia \\ ${ }^{4}$ Department of Applied Mathematics, Research School of Physics and Engineering, Australian National University, Canberra \\ ACT 2601, Australia
}

\begin{abstract}
In this paper, we report experimental results on granular compaction in a model system made of mono- and bidisperse ellipsoidal packings as well as sand packings with grain size polydispersity. The packings are subject to vertical tapping of varying duration (number of taps) and their internal three-dimensional structure is obtained using x-ray computed tomography. Particles positions and orientations are reconstructed and the global packing densities are computed. The analysis of the vertical and horizontal local packing fraction profiles reveal a homogeneous densification in the ellipsoidal packings, however, sand packings exhibit radial density gradient, possibly linked to the onset of convection.
\end{abstract}

\section{Introduction}

Granular materials are large conglomerations of discrete macroscopic particles. Yet despite this seeming simplicity, a granular system exhibits complex behaviours, such as size segregation [1], dilatancy [2], creep [3, 4], glassy behaviour [5, 6], shear banding [7] and pattern formation $[8,9]$. There are a number of factors that are responsible for this rich diversity; chief amongst them are: grain-grain friction, grain size distribution and particle shape ${ }^{1}$. Friction introduces a sharp nonlinearity in grain contact laws resulting in complex energy dissipation mechanisms. Particle shape and size distribution are two other primary parameters that determine granular properties at both microand macro-scale.

One particular physical phenomenon that has fundamental scientific value as well as outstanding industrial applications is the process of granular compaction. Understanding the connection between granular compaction and the slow relaxation of such out-of-equilibrium a-thermal systems [10], is one of the main themes of the field of Soft Matter $[11,12]$. On the application side; due to the fact that granular compaction results in porosity reduction (increase in packing density) and permeability changes, it has become the research focus for pharmaceutical and transport industries. In geomechanics and carbon storage,

\footnotetext{
${ }^{\star}$ e-mail: fabian.schaller@fau.de

$\star \star$ e-mail: g.schroeder-turk@murdoch.edu.au

$\star \star \star$ e-mail: mohammad.saadatfar@anu.edu.au

${ }^{1}$ Assuming rigid particles.
}

granular compaction and deformation of soil and rocks are the key mechanisms in predicting landslides [13] and long term safety of carbon storage [14].

Characterisation of compaction process at the grain scale is complex due to the addition of the time dimension. As the compaction takes place in a granular medium, the structure settles down in a permanent but metastable static configurations. A large part of the problem arises from the strongly nonlinear contact law between continually rearranging rigid bodies coupled with the dynamical nature of the contact network. In this context, the spatial distribution and temporal evolution of the free volume available to individual particles is a salient quantity that expands from micro- (local Voronoi volume) to macro- (global packing density) scales.

Microscopic (grain-scale) models for granular materials need to incorporate physical parameters that are pertinent at the particle scale such as friction coefficients, compressibility, etc. They also need to take into account distribution properties such as the variability in grain size and shape. In this paper, we aim to reduce the model complexity to essential ingredients by identifying the key mechanisms at play that govern the granular compaction. The key question we aim to address is the extension of the 'zero-th order' model -the spherical case- to model with first- and second-order complexity. Recent years have seen many studies addressing the effects of the particle shape while maintaining uniform particles. Ellipsoids [15, 16], tetrahedra and polyhedra, pear-shaped particles, etc. have all been investigated. There has also been substantial in- 


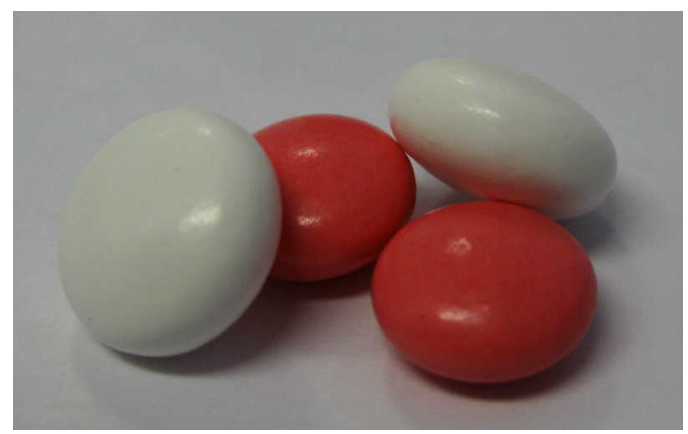

Figure 1. Pharmaceutical placebo pills with two different aspect ratios

vestigations into physical properties [15] that specifically change the compressibility [17].

Here, we focus on a different effect, namely the the compaction of bidisperse ellipsoidal \& spherical particles via tapping. In addition, we study compaction in sand packings to better understand the effect of particle shape irregularity. We investigate the effect of mixtures of ellipsoids of identical aspect ratio but different size. Evidently, the question of particle size distribution has been addressed before in systems of spherical particles, specifically in the context of glass forming systems [18, 19], as well as in the Apollonian packings [20,21]. Also, in studies of two-dimensional glass forming systems, bidispersity is a very commonly used tool to prevent crystallisation. However, the effect of size polydispersity of ellipsoidal packings is not extensively documented in the literature, even though it may have strong repercussions e.g. for the Voronoi cell distributions and hence local packing fractions.

Our results have practical applications in domains such as pore description in soil- and geosciences [22], which are crucial for understanding natural systems mechanical stability and flow permeability. Moreover, at a fundamental level, our results provide an opportunity to better our understanding of the non-equilibrium physics of compacting granular assemblies.

\section{Experiment}

To study the properties of bidisperse ellipsoid packings, we use two types of pharmaceutical placebo pills with an aspect ratio $\alpha \approx 0.57$ and large axis lengths of $8.9 \mathrm{~mm}$ and $10.2 \mathrm{~mm}$, as shown in figure 1 .

We start the preparation of the packing by poring the particles through a funnel into a cylindrical Perspex container of $144 \mathrm{~mm}$ diameter, as shown in figure 1. A set of steel wire grids has been placed at the bottom of the container before the fill. The circular grids have a square mesh size of $\approx 20 \mathrm{~mm}$, spatially separated by $\approx 25 \mathrm{~mm}$ in height and their mesh orientation is rotated by 45 degrees with respect to each other. During the filling process, the container is placed on a motorised, slowly rotating platform $(\approx 0.2 \mathrm{~Hz})$ to create a homogeneous and isotropic packing. The grid is slowly pulled out to loosen packing before the

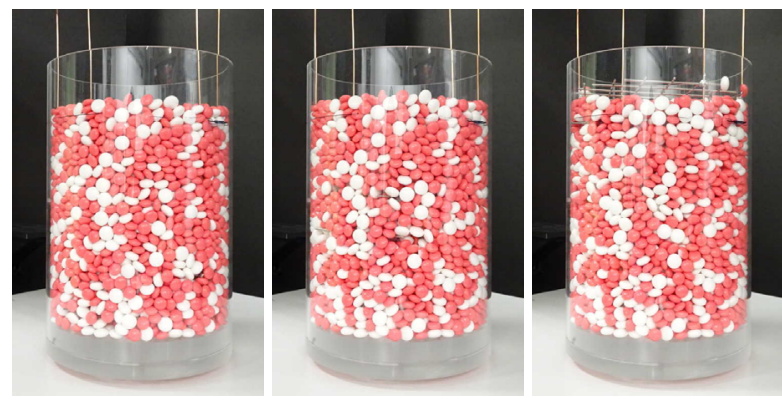

Figure 2. Loose packing preparation

experiments. As shown in figure 3, this technique leads to a reproducible initial condition with a global packing fraction of $0.655 \pm 0.002$. This preparation method is similar to the technique used for the study of monodisperse ellipsoidal particles [15].

The container is subsequently mounted atop an electro-dynamic shaker (TIRA TV51140). This $3 \mathrm{kN}$ shaker has a comparatively large diameter, 300mm armature which provides excellent lateral stability. The acceleration at the container base is measured using an accelerometer (B\&K 4507, 1000mV/g) via the shaker systems motion controller (Vibration Research, VR9500). The packings are now compacted by applying sinusoidally shaped pulses to the shakers linear amplifier. The chosen peak acceleration of $2 g$ (where $\left.g=9.81 \mathrm{~m} / \mathrm{s}^{2}\right)$ is higher than the earth's gravitational acceleration to allow the particles to move and rearrange [10]. The pulse width is 50 ms with a repetition rate of $3 \mathrm{~Hz}$. By varying the number of taps from 0 up to 1,000,000, packings of different densities are created, see figure 3. Each experiment (dataset) starts with a new initial preparation. Packings of 0 taps (initial loose packings), 100 taps and 1,500 taps are repeated several times to assure consistency. The variation in packing fraction is less than $0.5 \%$ for the initial packings reduces to $0.05 \%$ for 1500 taps.

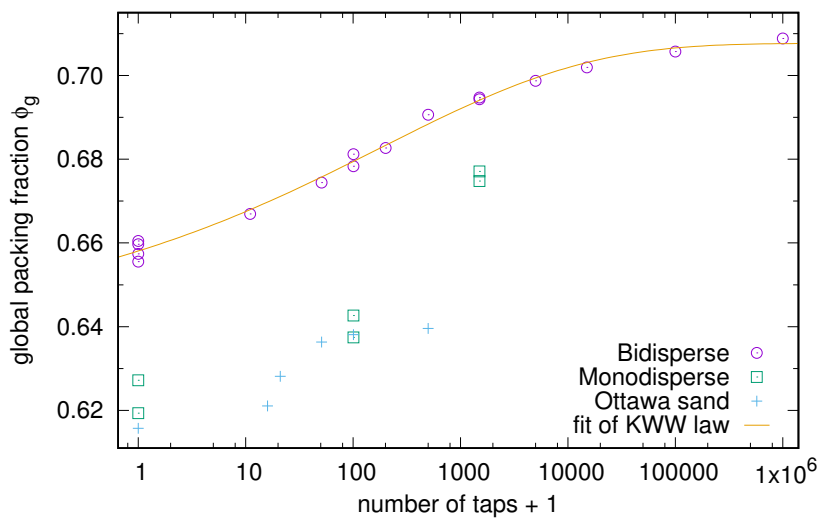

Figure 3. Number of taps vs. global packing fraction. Data of monodisperse and bidisperse ellipsoids is shown, as well as data of Ottawa sand packings. The orange curve is the best fit of the KWW law, see equation 1. 

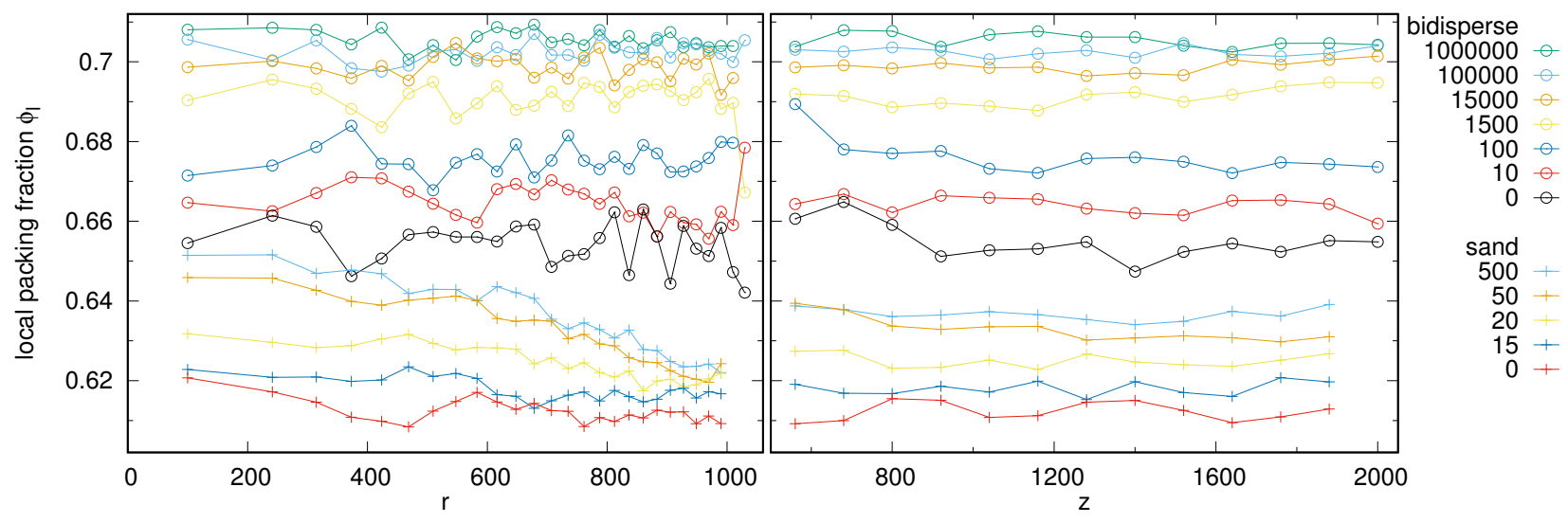

Figure 4. Radial and hight profile of the packing fraction for tapped packings of bidisperse ellipsoids and Ottawa sand. $r$ is the radial distance from the rotation axis of the container and $z$ the height from the bottom of the container in voxel units. Voxel size is $56 \mu \mathrm{m}$ for ellipsoids and $9 \mu \mathrm{m}$ for sand. The local packing fraction is averaged over all particles in each bin.

The resulting packings are imaged by helical x-ray tomography [23-25] and the particles positions and orientations are detected in the reconstructed image by a method based on a watershed algorithm [26, 27]. Particles close to the boundaries (cylinder walls, top and bottom) are removed from the analysis. The resulting packing is checked for spatial homogeneity in packing density. As a second dataset we show packings of Ottawa sand. The diameter of the sand grains is between 500 and $1000 \mu \mathrm{m}$. Loose sand packings are prepared in the same way as the bidisperse ellipsoid packings, by pouring the particles in a cylindrical Perspex container of $24 \mathrm{~mm}$ diameter and pulling a double grid through the packing to loosen it. The packings are again compacted by sinusoidal taps with peak acceleration of $2 \mathrm{~g}$. Figure 3 shows the global packing fraction vs. the number of applied taps for the bidisperse ellipsoid packing and the packings of Ottawa sand. As a reference, we include data for packings of monodisperse ellipsoids with approx. the same aspect ratio $\alpha=0.59$ prepared with a similar preparation method, which was published elsewhere [15].

\section{Results}

Figure 3 indicates that both systems show densification and density saturation, however, the ellipsoidal packing shows a very slow density saturation with a gentle change of densification starting at $\approx 10$ taps and another transition to density saturation at $\approx 1,000$ taps. The sand packing, however, exhibits a relatively sharp transition from the onset of densification ( $\approx 15$ taps) to density saturation at $\approx 50$ taps. The slow densification in the ellipsoidal packings is reminiscent of relaxation and compaction dynamics in glassy systems, which are commonly fitted by a stretched exponential law, the KWW (Kohlrausch, Williams, Watts) law [10, 12]

$$
X_{\mathrm{inf}}-\left(X_{\mathrm{inf}}-X_{0}\right) * \exp \left[-(\# \operatorname{taps} / \tau)^{\beta}\right]
$$

Figure 3 shows a fit of the KWW law to our data of bidisperse ellipsoids, which is in very good agreement. In con- trast, the global packing density in the sand packing, however, fully saturates at $\approx 50$ taps.

To better understand this sharp contrast in the behaviour of global packing density, we compute radial (edge to centre) and vertical (direction of gravity) density profiles in these systems by using Set Voronoi diagrams [28]. The local packing fraction of a particle is defined as the ratio between particles volume and its Voronoi cell. Fig. 4 shows the radial and vertical density profile of selected packings. The plots show that unlike the ellipsoidal packing, the radial density profile of the sand packing is inhomogeneous and the density monotonically decreases towards the middle of sand packing. This is consistant with our observation of sand pile-up in the middle of sand packings after 15 taps. Both ellipsoidal and sand packings exhibit homogeneous vertical density profiles.

\section{Conclusions and future work}

We have shown that using a "double-grid" mesh system combined with pouring grains into a rotating container, we are able to generate reproducible loose (initial state of tapping) of bidisperse ellipsoid and sand packings. The packings are then subject to a fixed ( $2 \mathrm{~g}$ ) tapping with varying number of taps. We compute the local and global packing density after each tapping stage. The ellipsoidal packings densify very slowly with the number of taps without showing any sign of structural inhomogeneity. The sand packings, however, exhibit sharp density-increase only after 15 taps and the global packing density saturates at 50 taps. By analysing the radial and vertical density profile of the sand packings, we find strong structural inhomogeneity first appearing at 15 taps and increasing up to 50 taps. The radial density inhomogeneity combined with our observation of the formation of sandpile in the middle of the packings suggests that convection may be at play inside the sand packings.

In sand packings, compaction slows down to almost full saturation very quickly and we also observe convection. A possible reason for it is the frictional forces; be- 
tween the particles and the container walls, which are more crucial because the sand particles are much smaller and lighter than the placebo ellipsoids. We are currently studying the effect that container roughness migh have on the onset of convection and the compaction of sand packings. Another future direction is to study the effect of friction by using ellipsoids of the same size and shape but different materials. Furthermore, we aim to investigate particle size segregation by varying experimental parameters.

\section{Acknowledgements}

We thank Weimer Pharma $\mathrm{GmbH}$ for providing the placebo pills. FMS acknowledges funding by the German Science Foundation (DFG) through the research group "Geometry and Physics of Spatial Random Systems" under Grant No. SCHR-1148/3-2 and. FMS acknowledges funding by Marcelja Fellowship at ANU. MS thanks Tim Senden for his continued support.

\section{References}

[1] A. Kudrolli, Reports on progress in physics 67, 209 (2004)

[2] O. Reynolds, 12 (1886)

[3] H.M. Jaeger, C.h. Liu, S.R. Nagel, Phys. Rev. Lett. 62, 40 (1989)

[4] J.A. TenCate, E. Smith, R.A. Guyer, Phys. Rev. Lett. 85, 1020 (2000)

[5] P. Charbonneau, J. Kurchan, G. Parisi, P. Urbani, F. Zamponi, Nature communications 5 (2014)

[6] A. Seguin, O. Dauchot, Phys. Rev. Lett. 117, 228001 (2016)

[7] M. Saadatfar et al. (2012)

[8] G.H. Ristow, Pattern formation in granular materials, 164 (Springer Science \& Business Media, 2000)

[9] N. Francois, M. Saadatfar, R. Cruikshank, A. Sheppard, Physical Review Letters 111, 148001 (2013)

[10] P. Philippe, D. Bideau, EPL (Europhysics Letters) 60, 677 (2002)

[11] M. van Hecke, Journal of Physics: Condensed Matter 22, $033101(2010)$
[12] J.B. Knight, C.G. Fandrich, C.N. Lau, H.M. Jaeger, S.R. Nagel, Phys. Rev. E 51, 3957 (1995)

[13] K. Hutter, Avalanche dynamics (Springer, 1996)

[14] S. Holloway, Energy Conversion and Management 38, S241 (1997)

[15] F.M. Schaller, M. Neudecker, M. Saadatfar, G.W. Delaney, G.E. Schröder-Turk, M. Schröter, Phys. Rev. Lett. 114, 158001 (2015)

[16] F.M. Schaller, S. Kapfer, J. Hilton, P. Cleary, C.D.M. K. Mecke, T. Schilling, M. Saadatfar, M. Schröter, G. Delaney, G. Schröder-Turk, EPL (Europhysics Letters) 111, 24 (2015)

[17] M. Saadatfar, A.P. Sheppard, T.J. Senden, A.J. Kabla, Journal of the Mechanics and Physics of Solids 60, 55 (2012)

[18] M. Maiti, S. Sastry, The Journal of chemical physics 141, 044510 (2014)

[19] D. Fiocco, G. Foffi, S. Sastry, Physical Review E 88, 020301 (2013)

[20] R.M. Baram, H. Herrmann, N. Rivier, Physical review letters 92, 044301 (2004)

[21] D. Stäger, H. Herrmann, arXiv preprint arXiv:1607.08391 (2016)

[22] A. Sufian, A.R. Russell, A.J. Whittle, M. Saadatfar, Granular Matter 17, 727 (2015)

[23] A. Katsevich, SIAM Journal on Applied Mathematics 62, 2012 (2002)

[24] T. Varslot, A. Kingston, G. Myers, A. Sheppard, Medical Physics 38, 5459 (2011)

[25] A. Sheppard et al., Nuclear Instruments and Methods in Physics Research Section B: Beam Interactions with Materials and Atoms 324, 49 (2014)

[26] M. Saadatfar, A.N. Shepard, M.K. Knackstedt, Advances in X-ray Tomography for Geomaterials pp. 269-276 (2010)

[27] F.M. Schaller, M. Neudecker, M. Saadatfar, G. Delaney, K. Mecke, G.E. Schröder-Turk, M. Schröter, AIP Conference Proceedings 1542, 377 (2013)

[28] F.M. Schaller, S.C. Kapfer, M.E. Evans, M.J. Hoffmann, T. Aste, M. Saadatfar, K. Mecke, G.W. Delaney, G.E. Schröder-Turk, Philos. Mag. 93, 3993 (2013) 